\title{
Metformin And Dichloroacetate Combination Exert A Synergistic Effect On Cell Viability Of Oral Squamous Cell Carcinoma
}

\author{
Seniz INANC ${ }^{1}$, Didem KELES ${ }^{2,5}$, Gorkem ESKIIZMIR ${ }^{3}$, Yasemin BASBINAR ${ }^{4}$, Gulgun OKTAY ${ }^{2}$ \\ ${ }^{1}$ Dokuz Eylul University, 1Institute of Health Sciences, ORCID: 0000-0002-4985-9022 \\ ${ }^{2}$ School of Medicine, Department of Medical Biochemistry, Didem KELES ORCID: 0000-0001-7350-8049 \\ Gulgun OKTAY ORCID: 0000-0002-0481-6029 \\ ${ }^{3}$ Manisa Celal Bayar University, Faculty of Medicine, Department of Otolaryngology-Head and Neck Surgery, Manisa - TURKEY \\ ORCID: 0000000231258288 \\ ${ }^{4}$ Institute of Oncology, Department of Translational Oncology, 35340 Inciralti, Izmir - TURKEY ORCID: 0000-0001-9439-2217 \\ ${ }^{5}$ Izmir Univ. of Economics, Vocational School of Health Services, Dept. of Medical Laboratory Techniques, 35330 Balcova, Izmir-TURKEY
}

\begin{abstract}
Objective: To assess the effects of Metformin, Dichloroacetate (DCA) and their combination on cell viability in oral squamous cell carcinoma, UPCI-SCC-131 cell line.
\end{abstract}

Methods: UPCI-SCC-131 cells were plated in 96 E-plate $(1 \times 104$ cells/well) and were treated with Metformin (1-16mM) and/or DCA (15-120mM) for 24-48-72h. xCELLigence SP system was used to monitor real time cell viability. In addition, drug combination index was analyzed with CompuSyn software according to Chou-Talalay method.

Results: Half-maximal inhibitory concentrations $\left(\mathrm{IC}_{50}\right)$ of Met- formin and DCA were found to be $3 \mathrm{mM}$ and $23 \mathrm{mM}$, respectively, for 72 hours. $\mathrm{Cl}$ values $(0.76-0.80)$ in all combination groups below 1 indicated that Metformin/DCA combination had a moderate synergistic effect on cell viability in UPCI-SCC-131 cells.

Discussion: Metformin/DCA combination synergistically decreased the cell viability of UPCI-SCC-131 cells. Therefore, a combined application of Metformin and DCA may be considered as a candidate therapy for the "drug repositioning" of the treatment of oral cavity cancer.

Keywords: Metformin, Dichloroacetate, drug combinations, oral cavity, cancer

\section{Introduction}

Oral squamous cell carcinoma (OSCC) is a predominant, aggressive and drug-resistant cancer type among head and neck cancers. Due to limitations of conventional therapy approaches; developing a novel and an effective therapeutic strategy is of utmost importance for the treatment of this cancer.

Metformin (1,1-dimethylbiguanide hydrochloride) is an antidiabetic agent which used in the treatment of Type $2 \mathrm{di}$ abetes and inhibits mitochondrial Complex I also known as $\mathrm{NADH} / \mathrm{ubiquinone}$ oxidoreductase. Currently, metformin has also found to be associated with improved survival in Type 2 diabetic patients with breast, prostate, colorectal, and head and neck cancer. ${ }^{[1]}$ Several studies have reported that metformin had a synergistic effect on the efficacy of chemotherapeutic approaches in several tumors, therefore, has great potential as a neoadjuvant anti-cancer agent. ${ }^{[2,3]}$

Dichloroacetate (DCA), a small kinase inhibitor of pyruvate dehydrogenase kinase (PDK), promotes the flux
Correspondence: Prof. Dr. Gulgun OKTAY

Dokuz Eylul University, School of Medicine, Department of Medical Biochemistry, 35340 Inciralti, Izmir - TURKEY e-mail: gulgun.oktay@deu.edu.tr

Received: 14.03.2019; Accepted: 14.05.2019
Online available at: www.entupdates.org

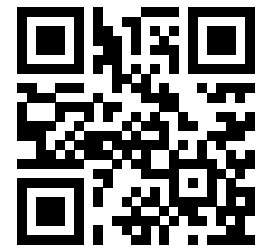


of carbohydrates into mitochondria through pyruvate dehydrogenase $(\mathrm{PDH})$ activation. Dichloroacetate exerts anti-tumorigenic functions via inhibition of PDK leading to altered energy metabolism and increased cell death in many tumors, including pancreatic, breast, endometrial and ovarian cancers. ${ }^{[4,5]}$ In addition, in vitro studies have indicated that DCA sensitized metformin cytotoxicity by reprogramming of energy metabolism in cancer cells. ${ }^{[6]}$

In the treatment of oral cavity cancer, new combination strategies that not only reduce cancer cell viability but also have low side effects are urgently required. Considering the therapeutic potential of metformin and DCA, the main objective of the study was to evaluate whether both of these drugs had a synergistic effect on the cell viability of UPCI-SCC-131 cells and contribute to combination therapy for the treatment of OSCC.

\section{Methods}

\section{Cell Culture}

UPCI-SCC-131 (Oral squamous cell carcinoma cell line), was purchased from DSMZ (Braunschweig, Germany). Cells were cultured in MEM supplemented with $10 \%$ fetal bovine serum (FBS) (Biochrom, Berlin, Germany), 1\% nonessential amino acids (Thermo Fisher Scientific, Waltham, MA, USA), $2 \mathrm{mM}$ L-Glutamine (Thermo Scientific, Waltham, MA, USA), and 100 units/mL penicillin/100 $\mu \mathrm{g} / \mathrm{mL}$ streptomycin (Thermo Fisher Scientific, Waltham, $\mathrm{MA}, \mathrm{USA}$ ) at $37^{\circ} \mathrm{C}$ in $5 \% \mathrm{CO}_{2}$ humidified incubator.

\section{Real Time Cell Viability Analysis}

UPCI-SCC- 131 cells were seeded at $1 \times 10^{4}$ cells/well into gold-coated 96 E-plate overnight and exposed to Metformin and DCA at various concentrations for 72 hours at $37^{\circ} \mathrm{C}$. The cell viability was monitored by using Real-Time Cell Analyzer (RTCA) Single Plate (SP) (Roche Applied Science, Mannheim, Germany and ACEA Biosciences, San Diego, CA, USA). The cell index value that represents cell viability was measured according to the relative change in electrical impedance, and also calculated by using the RTCA Software Version 2.0.

\section{Combination Index Calculation}

Drug combination was evaluated based on the principle of Chou and Talalay ${ }^{[7]}$ which is used in the calculation of the inhibitory effect $(\mathrm{Fa})$, dose reduction index (DRI), and effect of Metformin and DCA. The synergistic effects of the combined treatments were analyzed with CompuSyn version 1.0 software (ComboSyn, Inc., Paramus, NJ, USA). $\mathrm{CI}$ values $<1$ indicate synergism, CI values $=1$ are additive and $\mathrm{CI}$ values $>1$ shows antagonism. In addition, DRI values above 1 imply a favorable dose reduction in the drug combination compared to the monotherapy.

\section{Statistical Analysis}

Half maximal inhibitory concentrations $\left(\mathrm{IC}_{50}\right)$ of Metformin and DCA were determined by GraphPad Prism 7.0 (San Diego, CA, USA). All data were represented as means \pm standard error (SEM). Group comparisons were evaluated using Two-way ANOVA test with Dunnett's Post Hoc test. ${ }^{*} \mathrm{p} \leq 0.05 ;{ }^{* * * *} \mathrm{p} \leq 0.0001$ were defined as statistically significant.

\section{Results}

\section{Cell viability and $\mathrm{IC}_{50}$ values in Metformin and DCA treated UPCI-SCC-131 cells}

The xCELLigence RTCA SP system monitors the realtime cell viability through determination of cell impedance which is expressed as cell index. Figure 1A and Figure 2A represent the time-dependent effects of Metformin and DCA on UPCI-SCC-131 cell index, respectively. While all treated doses of Metformin had no inhibitory effect on cell viability for 24 and 48 hours, we found a dose-dependent reduction with an $\mathrm{IC}_{50}$ of $3 \mathrm{mM}$ in UPCI-SCC-131 cells for 72 hours (Figure 1B).

In contrast to Metformin, DCA significantly decreased the cell viability in a dose- and a time-dependent manner. The $\mathrm{IC}_{50}$ value of DCA for 72 hours was $23 \mathrm{mM}$ in UPCISCC-131 cells (Figure 2B).

\section{The evaluation of Drug Synergism}

The combination of Metformin and DCA was assessed according to Chou-Talalay method. Two drugs were combined in a constant ratio $(1: 60)$ to calculate CI and DRI values using CompuSyn software. As shown in Table 1 , inhibitory effect $(\mathrm{Fa})$ values of $0.05,0.5,0.75,0.90,0.95$, and

0.097 were used to determine CI and DRI values. All CI values ranged from 0.76 to 0.81 that indicate the combination of Metformin and DCA had a moderate synergistic effect against OSCC cell viability (Figure $3 \mathrm{~A}$ and $3 \mathrm{~B}$ ). We also found that all DRI values were above "1" which allows favorable dose reduction either Metformin or DCA 
Inanc $S$ et al.

Table 1. Inhibitory Effect (Fa), Dose Reduction Index (DRI), and Combination Index (CI) that belong to Metformin and DCA combination in UPCI-SCC-131 cells.

\begin{tabular}{cccccc}
\hline Inhibitory Effect $(\mathrm{Fa})$ & $\begin{array}{c}\text { Combination } \\
\text { Index }(\mathrm{Cl})\end{array}$ & $\begin{array}{c}\text { Dose Metformin } \\
(\mathrm{mM})\end{array}$ & $\begin{array}{c}\text { Dose DCA } \\
(\mathrm{mM})\end{array}$ & $\begin{array}{c}\text { Dose Reduction Index } \\
\text { (DRI) Metformin }\end{array}$ & $\begin{array}{c}\text { Dose Reduction } \\
\text { Index (DRI) DCA }\end{array}$ \\
\hline 0.05 & 0.76708 & 0.77904 & 7.97766 & 8.94194 & 1.52614 \\
0.5 & 0.78211 & 3.15865 & 23.4912 & 11.5938 & 1.43707 \\
0.75 & 0.78994 & 5.32516 & 35.1484 & 12.7735 & 1.40519 \\
0.90 & 0.79885 & 8.97766 & 52.5905 & 15.0321 & 1.37401 \\
0.95 & 0.80551 & 12.8068 & 69.1725 & 15.7539 & 1.35320 \\
0.97 & 0.81053 & 16.4897 & 84.0666 & & \\
\hline
\end{tabular}

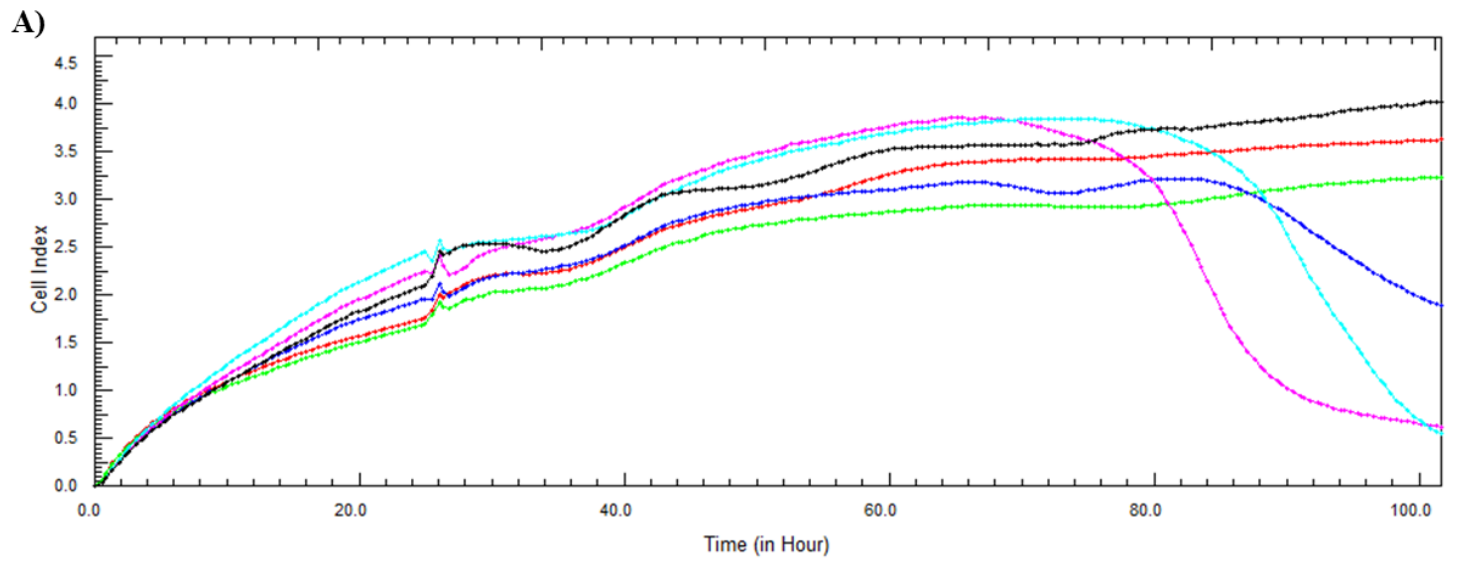

B)

\section{Metformin}



Figure 1. Cell index values and percentage of cell viability of Metformin-treated UPCI-SCC-131 cells. A) Dose-response curve of cell index was analyzed using the XCELLigence RTCA SP system. Cells were exposed to MEM (black), $1 \mathrm{mM}$ (red), $2 \mathrm{mM}$ (green), $4 \mathrm{mM}$ (blue), $8 \mathrm{mM}$ (pink), and 16mM (light blue) Metformin for 72 hours. B) The percentage of cell viability was calculated by using cell index values in Metformin-treated UPCI-SCC-131 cells for 24,48 and 72 hours. Data are expressed as mean $\pm \operatorname{SEM}(n=3)$. ${ }^{*} p \leq 0.05 ; * * * * p \leq 0.0001$ 
A)

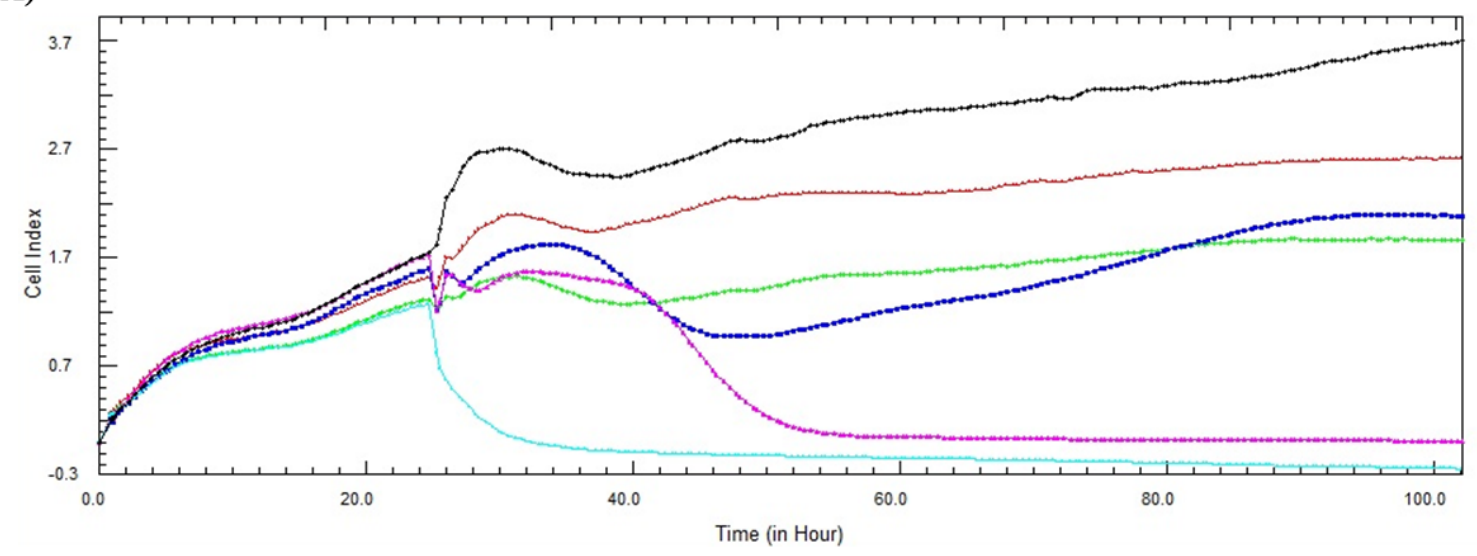

B)

\section{DCA}

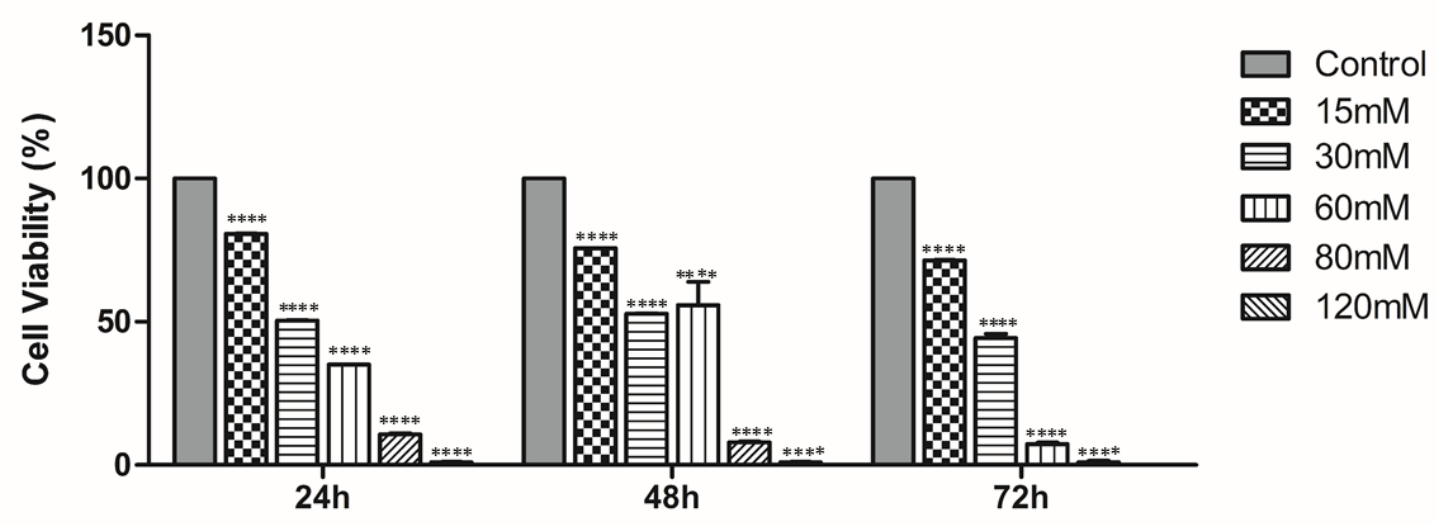

Figure 2. Effects of DCA on cell index and cell viability in UPCI-SCC-131 cells. A) Cell index values measured by xCELLigence RTCA SP system. Cells were treated with MEM (black), $15 \mathrm{mM}$ (red), $30 \mathrm{mM}$ (green), $60 \mathrm{mM}$ (blue), $80 \mathrm{mM}$ (pink), and 120mM (light blue) DCA. B) The percentage of cell viability was determined by using cell index values in DCA-treated UPCI-SCC-131 cells for 24,48 and 72 hours. Data are expressed as mean \pm SEM ( $n=3)$.

$\star * * * p \leq 0.0001$

\section{Discussion}

Oral squamous cell carcinoma is the sixth most common cancer and increasing rapidly worldwide. ${ }^{[8]}$ The current conventional strategies for the treatment of OSSC include surgical resection, radiotherapy and/or cisplatin based chemotherapy. ${ }^{[9]}$ Even though these multimodal strategies are effective for early stage-oral cavity cancer, 5-years survival rates are significantly lower for the patients with advanced stage-oral squamous cell carcinoma. Therefore, new FDA-approved drugs that lead to cytotoxicity in oral cavity cell carcinoma are needed to be addressed. In oncology, "drug repositioning" defines re-investigating the FDA-approved drugs for new therapeutic options particularly for cancer patients; thereby providing drug discovery that has minimal and known side effects, good bioavailability and low cost.

Recent studies have showed that the anti-diabetic drug, Metformin, inhibited the cell proliferation and induced cell death in several human cancers such as prostate cancer, ${ }^{[10]}$ 
A)

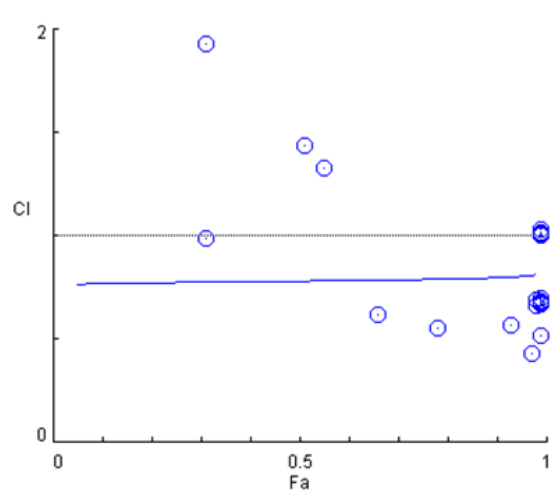

C)

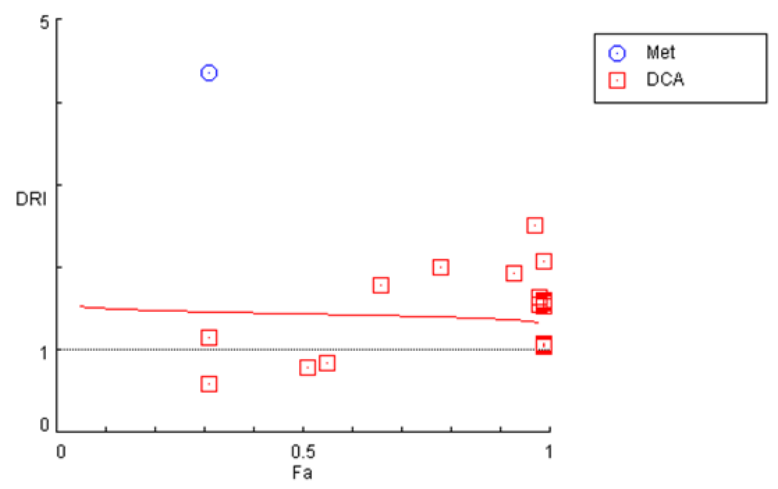

B)

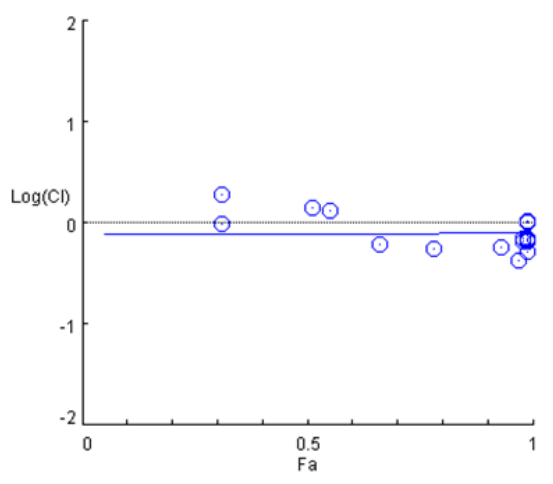

$\odot$ Met+DCA
D)

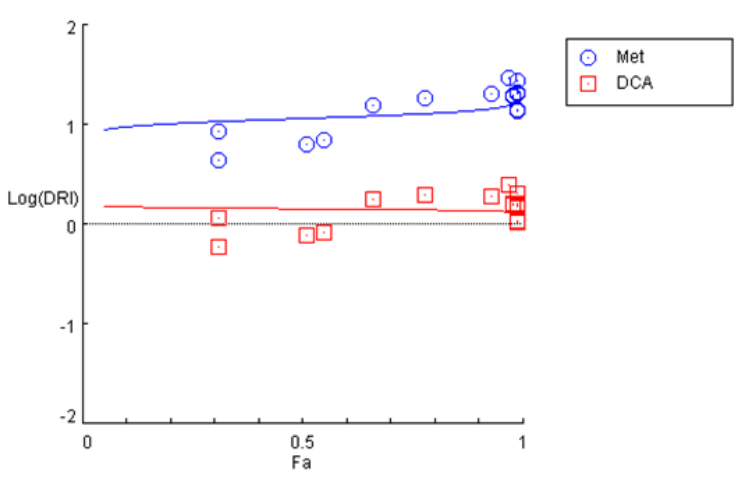

Figure 3. The combined effects of Metformin and DCA analyzed with CompuSyn system. A) Combination Index Plot (Fa-Cl plot) B) Logarithmic Combination Index Plot (Log(CI)-Fa) C) Dose Reduction Index Plot (Fa-DRI plot), and D) Logarithmic Dose Reduction Index Plot (Log(DRI)-Fa). Met; Metformin.

breast cancer, ${ }^{[11]}$ and OSCC. ${ }^{[12]}$ Moreover, metformin has a synergistic antitumor effect when used in combination with other well-known chemotherapeutic agents such as cisplatin, 5-fluorourasil, and paclitaxel. ${ }^{[13-15]}$

Dichloroacetate is widely used in the treatment of lactic acidosis, inhibits pyruvate dehydrogenase kinase (PDK) via phosphorylation of pyruvate dehydrogenase. It is reported that PDK-1 inhibition by DCA reversed Warburg effect, and this leads to a decreased cancer malignancy. Because of this role, DCA is a promising drug in the treatment of various cancers. ${ }^{[16]}$

In the current study, we investigated the effects of Metformin and DCA alone on cell viability and the potential of their combination against UPCI-SCC-131 cells. Our results reveal that Metformin and DCA inhibited the cell viability in time- and dose-dependent manner with an $\mathrm{IC}_{50}$ of 3 and $23 \mathrm{mM}$, respectively. The synergistic inhibition effects of Metformin and DCA which was analyzed with CompuSyn method, showed moderate synergism on cell viability of OSCC cells $(\mathrm{CI}<1)$. Similarly $\mathrm{Li}$ et al., reported that DCA and Metformin combination could inhibit the cell viability to as low as $50 \%$ in ovarian cancer cells. ${ }^{[17]} \mathrm{It}$ is also demonstrated that the combination of DCA and Metformin synergistically induce apoptosis via oxidative damage in breast cancer cells. ${ }^{[18,19]}$ Florio et al reported that both Metformin and DCA reduced cell viability in auton- 
onomic nervous system tumors, paragangliomas. In addition, DCA/Metformin/GW6471 combination showed strong synergistic effects on cell viability and clonogenic ability. [20]

The combination of drugs used in cancer therapy is expected to exhibit antitumorigenic behavior. In this regard,

\section{References}

1. Zhang ZJ, Li S. The prognostic value of metformin for cancer patients with concurrent diabetes: a systematic review and meta-analysis. Diabetes Obes Metab 2014;16:707-10.

2. Hanna RK, Zhou C, Malloy KM, et al. Metformin potentiates the effects of paclitaxel in endometrial cancer cells through inhibition of cell proliferation and modulation of the mTOR pathway. Gynecologic Oncology 2012;125:458-69.

3. Iliopoulos D, Hirsch HA, Struhl K. Metformin Decreases the Dose of Chemotherapy for Prolonging Tumor Remission in Mouse Xenografts Involving Multiple Cancer Cell Types. Cancer research 2011;71:3196-201.

4. Chen Y, Cairns R, Papandreou I, Koong A, Denko NC. Oxygen consumption can regulate the growth of tumors a new perspective on the Warburg effect. PLoS One 2009;4:e7033.

5. Rajeshkumar NV, Yabuuchi S, Pai SG, et al. Treatment of Pancreatic Cancer Patient-Derived Xenograft Panel with Metabolic Inhibitors Reveals Efficacy of Phenformin. Clin Cancer Res 2017;23:5639-47.

6. YW Choi, IK Lim. Sensitization of metformin-cytotoxicity by dichloroacetate via reprogramming glucose metabolism in cancer cells. Cancer Lett 2014;346:300-8.

7. Chou TC, Talalay P. Quantitative analysis of dose effect relationships: the combined effects of multiple drugs or enzyme inhibitors. Adv Enzyme Regul 1984;22:27-55.

8. Sasahira T, Kirita T. Hallmarks of Cancer-Related Newly Prognostic Factors of Oral Squamous Cell Carcinoma. Int J Mol Sci 2018;19 doi: 10.3390/ijms19082413.

9. Feller L, Lemmer J. Oral Squamous Cell Carcinoma: Epidemiology, Clinical Presentation and Treatment. J Cancer Ther 2012;3:263-8.

10. Chen X, Li C, He T, et al. Metformin inhibits prostate cancer cell proliferation, migration, and tumor growth through upregulation of PEDF expression. Cancer Biol Ther 2016;17:507-14.

11. Zhuang Y, Miskimins WK. Metformin induces both caspase-dependent and poly(ADP-ribose) polymerase-dependent cell death in breast cancer cells. Mol Cancer Res 2011;9:603-15. our results suggest that the potential use of metformin and DCA combination as an anticancer agent may be a promising therapeutic strategy for the treatment of oral cavity cancers. However, further experimental and clinical studies are required.

12. Guimarães TA, Farias LC, Santos ES, et al. Metformin increases PDH and suppresses HIF- $1 \alpha$ under hypoxic conditions and induces cell death in oral squamous cell carcinoma. Oncotarget 2016;7:55057-68.

13. Qi X, Xu W, Xie J, et al. Metformin sensitizes the response of oral squamous cell carcinoma to cisplatin treatment through inhibition of NF- $\mathrm{kB} /$ HIF-1 $\alpha$ signal axis. Scientific Reports 2016;6:35788.

14. Harada K, Ferdous T, Harada T, Ueyama Y. Metformin in combination with 5-fluorouracil suppresses tumor growth by inhibiting the Warburg effect in human oral squamous cell carcinoma. Int J Oncol 2016;49:27684.

15. Hanna RK, Zhou C, Malloy KM, et al. Metformin potentiates the effects of paclitaxel in endometrial cancer cells through inhibition of cell proliferation and modulation of the mTOR pathway. Gynecol Oncol 2012;125:458-69.

16. Li B, Zhu Y, Sun Q, et al. Reversal of the Warburg effect with DCA in PDGF-treated human PASMC is potentiated by pyruvate dehydrogenase kinase-1 inhibition mediated through blocking Akt/GSK-3 $\beta$ signalling. Int J Mol Med 2018;42:1391-400.

17. Li B, Li X, Ni Z, et al. Dichloroacetate and metformin synergistically suppress the growth of ovarian cancer cells. Oncotarget 2016;7:5945870.

18. Haugrud AB, Zhuang Y, Coppock JD, Miskimins WK. Dichloroacetate enhances apoptotic cell death via oxidative damage and attenuates lactate production in metformin-treated breast cancer cells. Breast Cancer Res Treat 2014;147:539-50.

19. Hong SE, Jin HO, Kim HA, et al. Targeting HIF-1alpha is a prerequisite for cell sensitivity to dichloroacetate (DCA) and metformin. Biochem Biophys Res Commun 2016;469:164-70.

20. Florio R, De Lellis L, Veschi S, et al. Effects of dichloroacetate as single agent or in combination with GW6471 and metformin in paraganglioma cells. Sci Rep 2018;8:13610.

This is an open access article distributed under the terms of the Creative Commons Attribution-NonCommercial-NoDerivs 3.0 Unported (CC BY- NCND3.0) Licence (http://creativecommons.org/licenses/by-nc-nd/3.0/) which permits unrestricted noncommercial use, distribution, and reproduc- tion in any medium, provided the original work is properly cited.

Please cite this article as: Inanc S, Keles D, Eskiizmir G, Basbinar Y, Oktay G. Metformin And Dicholoroacetate Combination Exert A Synergistic Effect On Cell Viability Of Oral Squamous Cell Carcinoma. ENT Updates 2019;9(2): 68-73 